\title{
ATLAS and ultra high energy cosmic ray physics
}

\author{
James Pinfold ${ }^{\mathrm{a}}$ \\ For the ATLAS Collaboration, Physics Department, University of Alberta, Edmonton, Alberta, Canada
}

\begin{abstract}
After a brief introduction to extended air shower cosmic ray physics the current and future deployment of forward detectors at ATLAS is discussed along with the various aspects of the current and future ATLAS programs to explore hadronic physics. The emphasis is placed on those results and future plans that have particular relevance for high-energy, and ultra high-energy, cosmic ray physics. The possible use of ATLAS as an "underground" cosmic muon observatory is briefly considered.
\end{abstract}

\section{Introduction}

Over the past ten years or so ATLAS has been involved in developing the detector coverage in the forward direction in order to expand the physics horizons of the ATLAS detector in the arena of hadronic physics. A discussion of recent developments in the forward detector system of the ATLAS detector will be presented in section one. In section two we will present the latest results in the ATLAS study of the p-p total, inelastic and elastic cross-section and in section three the latest $\mathrm{p}-\mathrm{p}$ charged particle distributions. In section four we will briefly consider some implications of ATLAS hadronic physics results for of high energy cosmic ray physics. Penultimately, in section five, we will touch on the possible use of the ATLAS detector as an underground cosmic muon observatory.

\subsection{High energy cosmic ray physics and hadronic physics at the LHC}

Understanding the sources and propagation of high energy cosmic rays are core issues of astroparticle physics. There is strong evidence that supernova remnants can accelerate cosmic rays up to energies of $\sim \mathrm{Z} \times 10^{14} \mathrm{eV}$ (where $\mathrm{Z}$ is the charge of the cosmic ray nucleus). However, the sources and accelerating mechanisms for particles with higher energies, up to $\sim 10^{20} \mathrm{eV}$ are not known [1]. Accelerating particles to such energies requires exceptional astrophysical objects and/or novel accelerating mechanisms [2]. Ultra-high energy cosmic rays are messengers from the extreme Universe that provide a window to particle physics at energies up to $\sqrt{(s)} \sim$ $400 \mathrm{TeV}$.

As can be seen from the energy spectrum of cosmic rays shown in Fig. 1 the study of cosmic rays of energies higher than about $10^{14} \mathrm{eV}$ is hampered by the low flux of the particles incident on the Earth. Thus, one has to use very large detector areas that use the upper atmosphere as a target and/or a calorimeter and resort to the large aperture observation of extensive air showers produced by cosmic rays entering the atmosphere [3]. The challenge to this approach is the need of detailed air shower simulations for

a e-mail: jpinfold@ualberta.ca deriving the primary particle energy and composition from measured air shower observables.

A compilation of more recent measurements of the flux of cosmic rays is given in Fig. 2. Measurements at the LHC allow us to access, for the first time, energies beyond the 'knee' in the cosmic ray spectrum where there is a sudden steepening of the power law of the flux at about $3 \times 10^{15} \mathrm{eV}$, which is not yet completely understood. Additionally, the very high energy cosmic ray flux exhibits an ankle at about $3 \times 10^{18} \mathrm{eV}$ (thought to be related to the transition between galactic and extragalactic cosmic rays $[4,5])$ and a very strong suppression at energies above $8 \times 10^{19} \mathrm{eV}$.

One possible cause of this cut off at the highest cosmic ray energies is GZK flux suppression [6], arising from scattering of ultra high energy cosmic primaries off $\mathrm{CMB}$ photons. However, it could be that the mass composition indicates that the upper end of the energy spectrum is more likely related to the maximum injection energy of the particles at the sources [7].

To study the sources of cosmic particles at energies above the knee and the astrophysical origin of the main features of the cosmic ray spectrum we need to derive the mass composition of cosmic rays. Thus, being able to reliably deduce the mass composition of cosmic rays from air shower measurements is of fundamental importance and is currently hampered mainly by the large uncertainties in predicting hadronic multi-particle production at high energy $[4,8]$. Thus, performing further measurements at accelerators is the only way to make progress.

Cosmic ray physics interaction models have been tuned to describe particular data sets taken at certain collider energies and also the energy dependence of multiparticle production. While cosmic ray models were developed before LHC was turned on, their predictions bracket many distributions measured in minimum-bias mode at LHC. This success in the development of the phenomenology developed for soft hadronic interactions can be improved considerably by tuning the models to match the LHC data. In the following document we will discuss LHC measurements that can further improve the understanding of hadronic multiparticle production with direct relevance to cosmic ray physics and air shower simulations. 


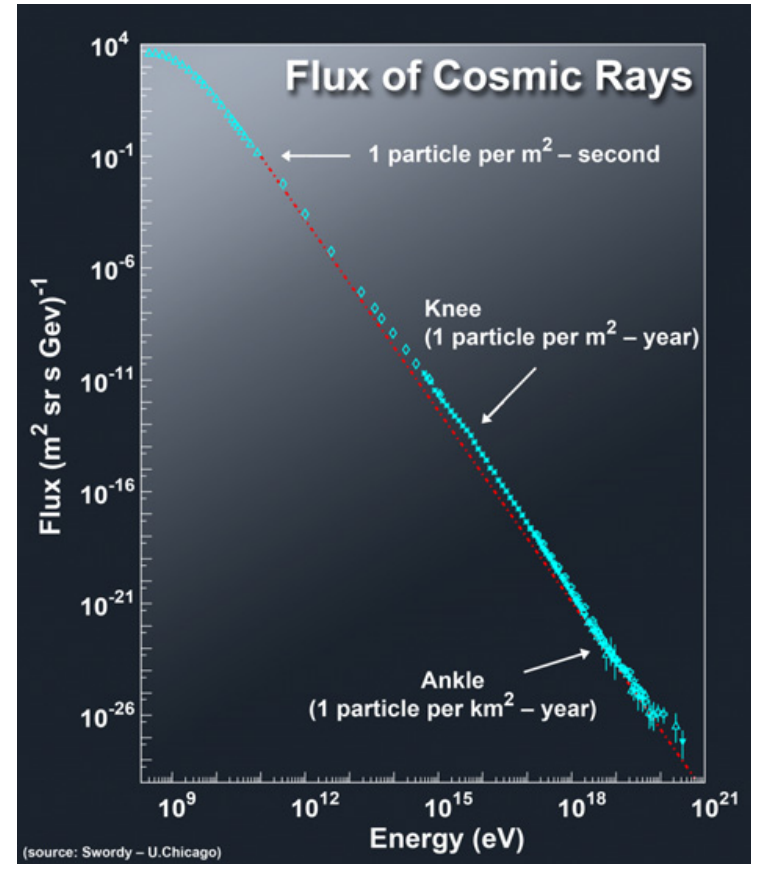

Figure 1. The cosmic ray energy spectrum.

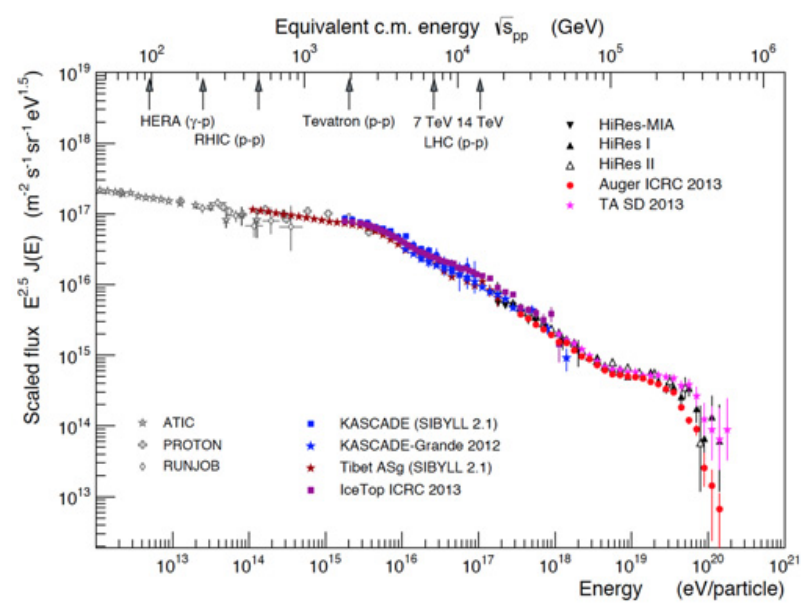

Figure 2. Compilation of representative measurements of the flux of cosmic rays at Earth (from [4], updated). The equivalent energies of LHC proton-proton collisions at different c.m.s. energies are shown at the upper axis.

\section{The ATLAS forward detector system}

The ATLAS Forward Detector system is shown in Fig. 3 indicating their distance from the ATLAS Interaction Point (IP) and the pseudorapidity $(\eta)$ range that they cover. In this report we will consider the following forward detectors: the New Minimum Bias Trigger Scintillator (MBTS) [9]; the Absolute Luminosity for ATLAS (ALFA) [10] and ATLAS Forward Protons (AFP) [11]. These detectors, in combination with the main ATLAS detector, provide the results discussed in this paper.

\subsection{The new MBTS detector}

The first Minimum Bias Trigger Scintillators (MBTS) delivered the minimum bias triggers for for the low luminosity LHC's Run I, from 2009-2013 for protonproton, lead-lead and lead-proton collisions. An upgraded

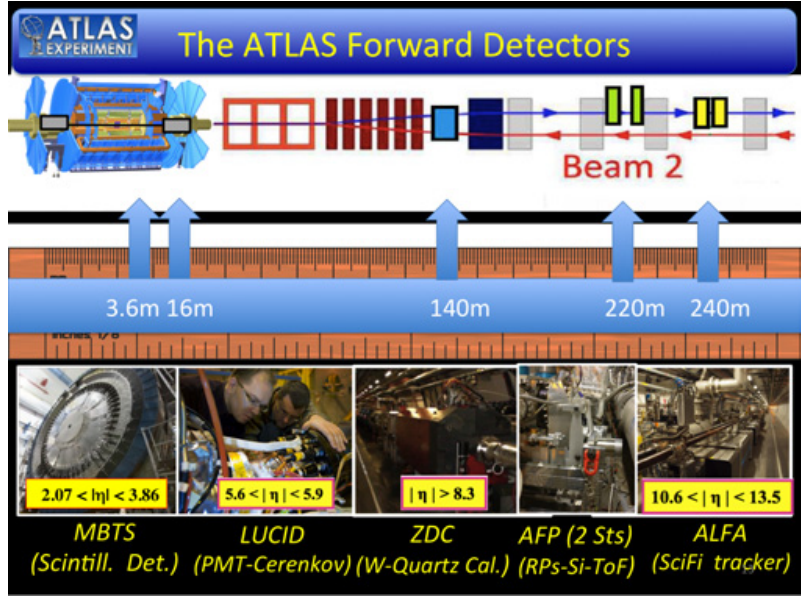

Figure 3. The Forward Detector system of the ATLAS experiment.

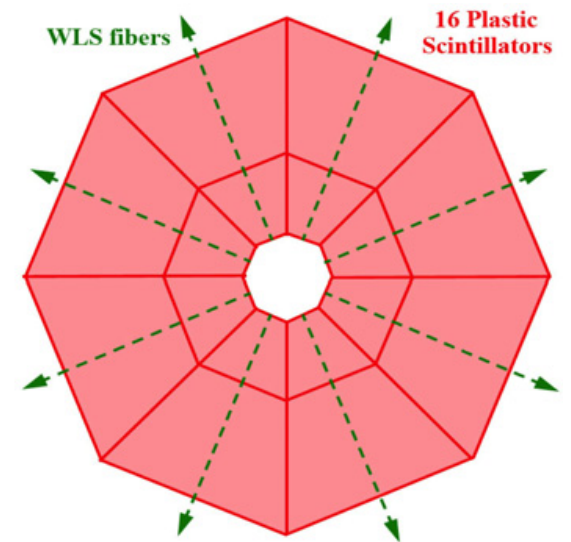

Figure 4. The basic structure of the Run I and Run II MBTS detector.

version of MBTS was installed for Run II of the LHC (2015-2018). This new MBTS will provide the key ingredients for the physics measurements at larger LHC collision energies of the charge multiplicity, proton-proton cross section, rapidity gap measurements, etc. The upgrade strategy included a replacement of radiation damaged scintillator and a modification of the read out scheme for Run II conditions.

It is estimated that in Run I, the MBTS received a dose in the range of 0.1 to $0.4 \mathrm{~Gy}$. Since in Run II, the same Soft QCD measurements have to be performed for the new LHC energy $\sqrt{s}=13 \mathrm{TeV}$, and at potentially higher luminosity, the decision was made to replace the MBTS with a new detector based on the existing design and trying to keep the same general structure and materials used in the Run 1 MBTS detector.

The basic structure of the MBTS is shown in Fig. 4. The readout scheme for Run II was modified in the following ways: the outer ring detectors light connectors have been merged two by two, while the inner rings have been kept as they were in Run I. The WLS fibres path has been modified and run closer to the detector edges, reducing the dips in the efficiency observed in Run I. The $\eta$ coverage has been slightly modified, with the outer and inner ring covering $2.08 \leq|\eta| \leq 2.76$ and $2.76 \leq|\eta| \leq$ 3.86 , respectively. Side A of the Run II MBTS detector was 


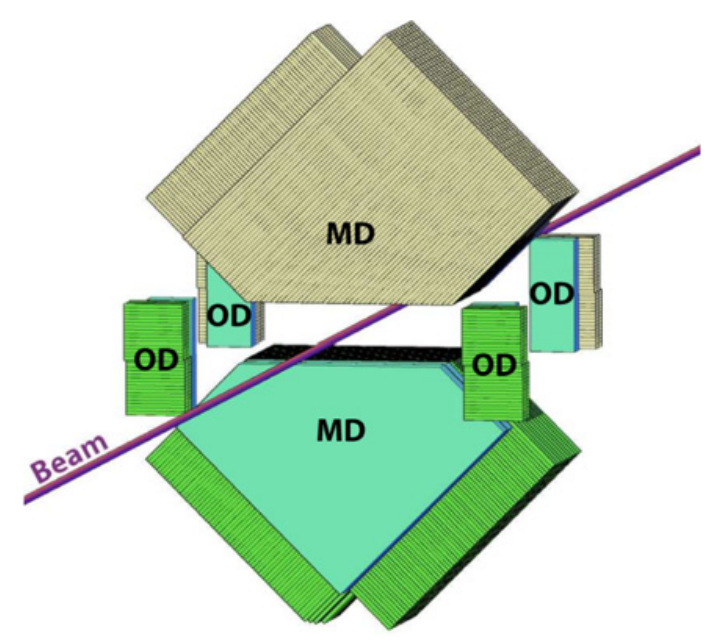

Figure 5. A sketch of the detector principle: MD labels the main detectors above and below the beam while OD stands for the overlap detectors. The lines across MDs and ODs indicate the orientation of the scintillating fibres. The areas without a fibre structure mark the trigger tiles.

installed in February 2014, while the C side was installed in July 2014.

\subsection{The ALFA detector}

The ALFA detector (Absolute Luminosity For ATLAS) is designed to measure elastic proton (small-angle) scattering. Two tracking stations are placed on each side of the central ATLAS detector at distances of $238 \mathrm{~m}$ and $241 \mathrm{~m}$ from the interaction point. The tracking detectors are housed in Roman Pots (RPs) which can be moved to within millimetres of the circulating proton beams. Combined with special beam optics, for example high $\beta$ allows the detection of protons at scattering angles down to $10 \mu \mathrm{rad}$. Each station has an upper and lower RP connected by bellows to the primary LHC vacuum. Elastically scattered protons are detected in the main detectors (MDs) while dedicated overlap detectors (ODs) measure the distance between upper and lower MDs. The arrangement of the upper and lower MDs and ODs, with respect to the beam, is illustrated in Fig. 5.

The ALFA tracking detectors are based on staggered layers of square cross-section scintillating fibres, read out by Multi-Anode Photo-Multiplier Tubes (MAPMTs). The fibres of the Main Detectors (MDs) are inclined at $45^{\circ}$ with respect to the vertical. The sensitive region has the shape of a truncated square. In the ODs the fibres are placed horizontally measuring the vertical coordinate only. Both, MDs and ODs, are equipped with scintillator tiles for triggering. They are made of $3 \mathrm{~mm}$ thick standard plastic scintillator and read out via bundles of clear plastic fibres of $0.5 \mathrm{~mm}$ diameter.

\subsection{The AFP detector}

The ATLAS Forward Proton (AFP) detector will identify events in which one or both protons emerge intact from the proton-proton collisions at the LHC. The installation of AFP is being performed in two stages: an AFP tracking system at one side of the IP, was already installed during the end-of-year 2015-2016 shutdown. The full two-arm

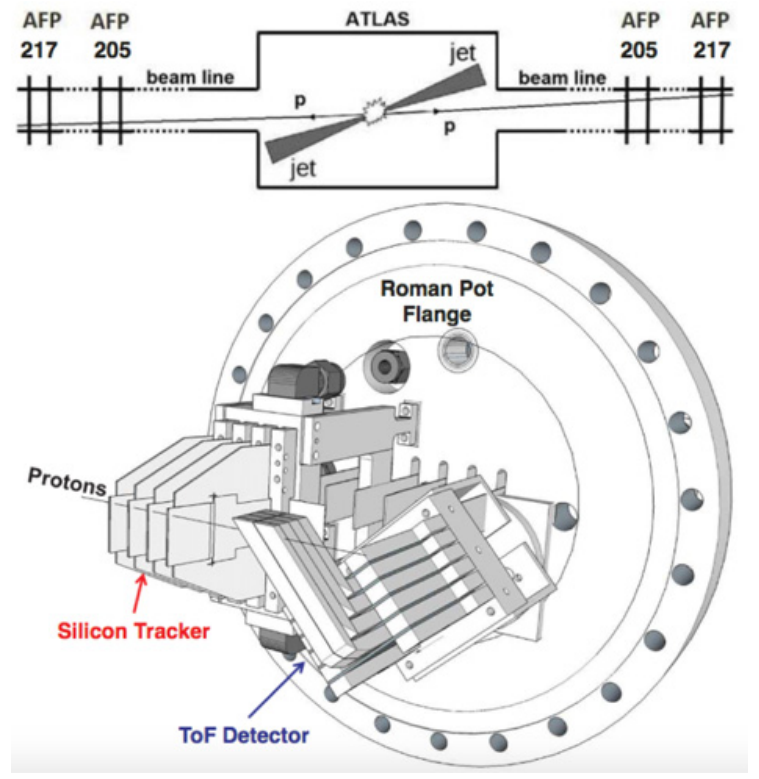

Figure 6. Layout of the AFP stations at both sides of the ATLAS IP at 205 and $217 \mathrm{~m}$. Bottom: Design of the $217 \mathrm{~m}$ AFP detector including tracking and time-of-flight systems (the final version will comprise four ToF LQbars per train).

system, with both tracking and timing detectors at both sides of the IP, is currently being completed during the extended end-of-year 2016-2017 shutdown. The final AFP detector will consist of two Roman-pot stations at $205 \mathrm{~m}$ and $217 \mathrm{~m}$, each side of the ATLAS IP, as depicted in Fig. 6 top.

Using a Roman Pot system, tracking and timing detectors can be placed 2-3 mm from the beam, $205 \mathrm{~m}$ and $217 \mathrm{~m}$ away from the ATLAS interaction point. The 3-D silicon tracker will provide momentum measurement, while the time of flight system is used to reduce the background from multiple proton-proton collisions. The study of soft and hard diffractive events at low luminosities $(\mu \approx 1)$ is the core of the AFP physics program.

\section{Total $p-p$ elastic, inelastic and total cross-sections}

A measurement of the total p-p cross section; elastic, inelastic and total cross-sections was performed with the ALFA sub-detector of ATLAS at the LHC at $\sqrt{s}$ of $7 \mathrm{TeV}$ and $8 \mathrm{TeV}$. In the case of the $8 \mathrm{TeV}$ measurement [12] an integrated luminosity of $500 \mathrm{fb}^{-1}$ was accumulated in a special run with high- $\beta^{*}$ beam optics to measure the differential elastic cross section as a function of the Mandelstam $t$. A fit to the differential elastic cross section in the $-t$ range from $0.014 \mathrm{GeV}^{2}$ to $0.1 \mathrm{GeV}^{2}$ was used to extrapolate to $t \rightarrow 0$. The measured differential crosssection and the fit of the theoretical prediction for that distribution is shown in Fig. 7. The total cross-section can be calculated using the Optical Theorem:

$$
\sigma_{t o t}=4 \pi \operatorname{Im}\left[f_{e l}(t \rightarrow 0)\right]
$$

which relates the total p-p cross-section $\sigma_{\text {tot }}$ to the elasticscattering amplitude extrapolated to the forward direction $f_{e l}(t \rightarrow 0)$, with $t$ being the four-momentum transfer 


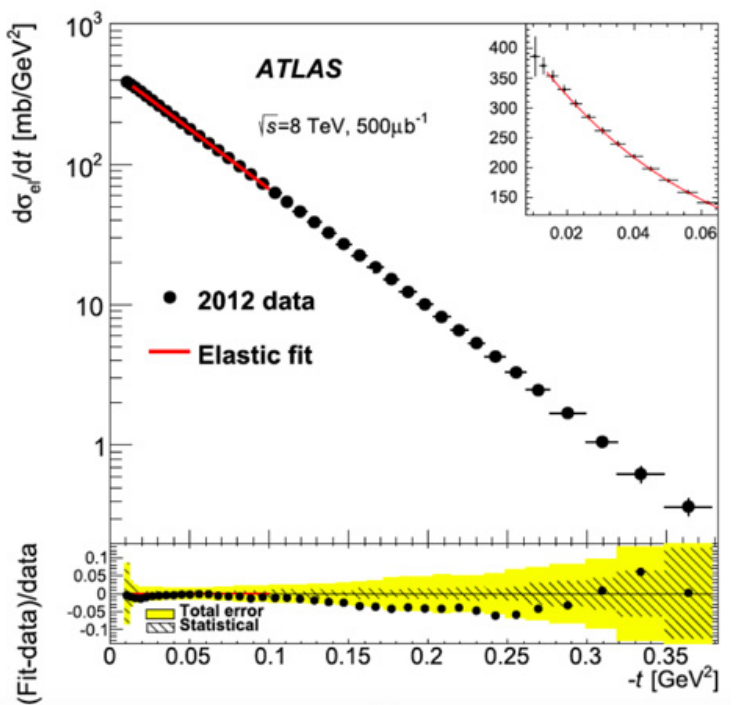

Figure 7. The fit of the theoretical prediction to the differential elastic cross section with $\sigma_{\text {tot }}$ and the slope of the exponential function describing the elastic cross section, B, as free parameters. In the lower plot the points represent the relative difference between fit and data, the yellow (light grey) area represents the total experimental uncertainty and the hatched area the statistical component. The red line (grey) indicates the fit range; the fit result is extrapolated in the lower plot outside the fit range. The upper right insert shows a zoom of the data and fit at small $t$.

squared. The result of the fit is:

$$
\begin{aligned}
\sigma_{\text {tot }}(p p \rightarrow X)= & 96.07 \pm 0.18(\text { stat. }) \\
& \pm 0.85(\text { exp. }) \pm 0.31(\text { extr. }) \mathrm{mb}
\end{aligned}
$$

where the first error is statistical, the second accounts for systematic uncertainties and the last arises from uncertainties in the extrapolation $t \rightarrow 0$. The measurements at $8 \mathrm{TeV}$ are significantly more precise than the previous measurements at $7 \mathrm{TeV}$ because of the smaller luminosity uncertainty and a larger data sample.

Using the total cross section, the total integrated elastic cross section, $\sigma_{e l}$, can be calculated, provided that the Coulomb amplitude is neglected, using the formula:

$$
\sigma_{e l}=\frac{\sigma_{t o t}^{2}}{B} \frac{1+\rho^{2}}{16 \pi \hbar^{2} c^{2}}
$$

The result is:

$$
\sigma_{e l}=24.33 \pm 0.04(\text { stat. }) \pm 0.39(\text { syst. }) \mathrm{mb}
$$

The total inelastic cross-section is determined by subtraction of the total elastic cross section from the total cross section. The resulting value is:

$$
\left.\left.\sigma_{\text {inel }}=71.73 \pm 0.15 \text { (stat. }\right) \pm 0.69 \text { (syst. }\right) \mathrm{mb}
$$

The measurements of ATLAS and TOTEM are compared to measurements at lower energy and to a global fit [13] in Fig. 8 for $\sigma_{\text {tot }}$.TOTEM also reported evidence of non-exponential behaviour of the differential cross-section [14]. The TOTEM Collaboration measured $\sigma_{\text {tot }}$ using the luminosity-independent method, using the same LHC fill used by ATLAS-ALFA. The result they obtained was $\sigma_{\text {tot }}=101.7 \pm 2.9 \mathrm{mb}$ [21], $1.9 \sigma$ higher than the ATLAS measurement presented here.

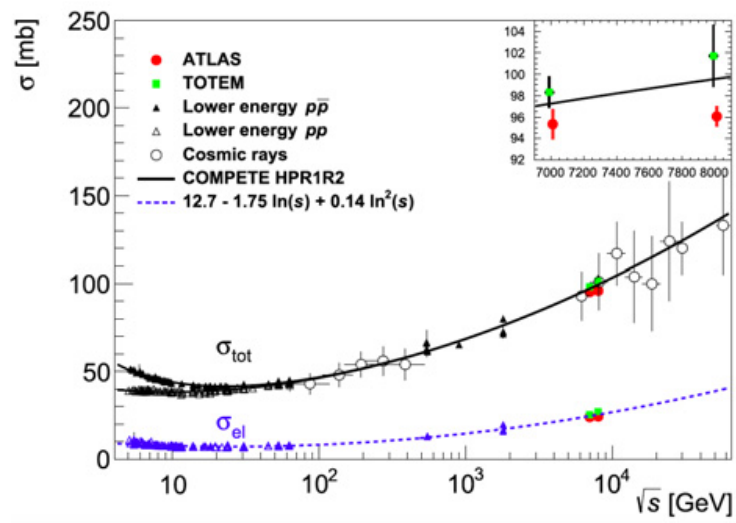

Figure 8. Comparison of total and elastic cross-section measurements presented here with other published measurements $[13,15-19]$ and model predictions as a function of the centre-ofmass energy.

\subsection{Measurement of the inelastic cross-section with the MBTS detector}

The inelastic proton-proton cross section was measured utilizing $60 \mu b^{-1}$ of p-p collisions at a center-of-mass energy of $13 \mathrm{TeV}$ with the ATLAS detector at the LHC [20]. Inelastic interactions were selected using the ATLAS MBTS detector $(2.07<|\eta|<3.86)$ with the trigger requirement that there is at least one MBTS hit above threshold. The counters are not sensitive to elastic pp scattering and diffractive dissociation processes in which neither proton dissociates into a system, $\mathrm{X}$, of mass $M_{X}>$ $13 \mathrm{GeV}$, or equivalently, $\xi=M_{X}^{2} / s>10^{-6}$. The crosssection measurement is reported in this fiducial region, $\xi>10^{-6}$, and after extrapolation to the total inelastic cross section using models of inelastic interactions.

Two sets of events passing the MBTS trigger requirements were selected: an inclusive set and a singlesided set. The inclusive selection demands at least two MBTS counters. Requiring two hits rather than one substantially reduces background due to collision-induced radiation and activation. To constrain the diffractive component of the cross-section and reduce the uncertainty in extrapolation to $\sigma_{\text {inel }}$, an additional single-sided selection is defined, requiring hits in at least two counters on one side of the detector and no hits on the other. In the data, 4,159,074 events pass the inclusive selection and 442,192 events pass the single-sided selection. A plot of the ratio of single sided events to inclusive events as a function of $f_{D}=\left(\sigma_{S D}+\Sigma_{D D}\right) / \sigma_{\text {inel }}$ is given in Fig. 9. To facilitate background studies, data were also collected with the same selection when no proton bunch ("empty") or a single proton bunch from only one of the two beams ("single beam") was passing through ATLAS.

A cross-section of $68.1 \pm 1.4 \mathrm{mb}$ is measured in the fiducial region $\xi=M_{X}^{2}>10^{-6}$, where $m_{X}$ is the larger invariant mass of any two final state hadronic systems separated by the largest rapidity gap in the event. In this range the MBTS detector is highly efficient. For diffractive events this corresponds to cases where at least one proton dissociates to a system with $M_{X}>13 \mathrm{GeV}$. The fiducial cross-section is determined from:

$$
\sigma_{\text {ineal }}^{\text {fid }}\left(\xi>10^{-6}\right)=\frac{N-N_{B G}}{\epsilon_{\text {trig }} \times \mathcal{L}} \frac{1-f_{\xi<10^{-6}}}{\epsilon_{\text {sel }}}
$$




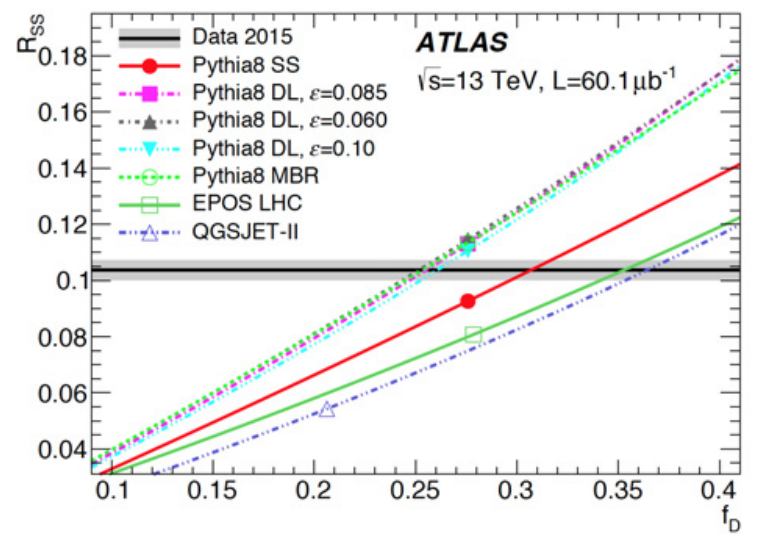

Figure 9. The ratio of the number of single-sided to inclusive events (RSS) as a function of the fraction of the cross section that is diffractive according to each model ( fD). The default value of $f_{D}$ in each model is shown with a marker

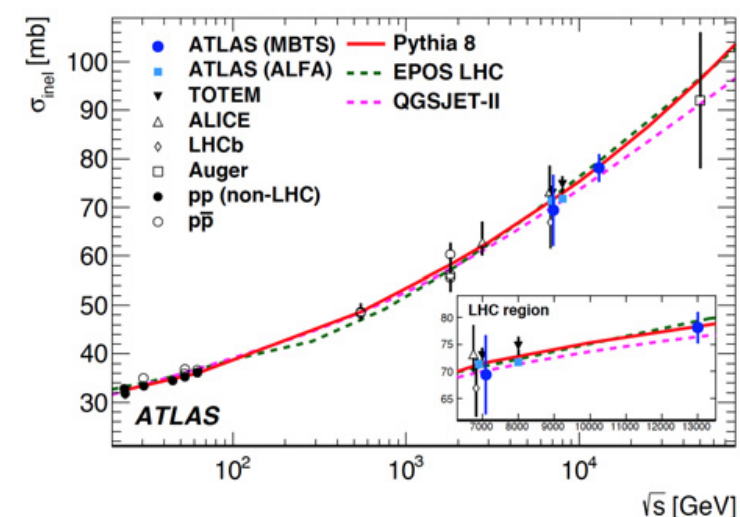

Figure 10. The inelastic proton-proton cross section versus $\sqrt{s}$. Measurements from other hadron collider experiments [13,15, 21-23] and the Pierre Auger experiment [16] are also shown. Some LHC data points have been slightly shifted in the horizontal position for display purposes. The data are compared to the Pythia8, Epos LHC and QGSJet-II MC generator predictions. The uncertainty in the ATLAS ALFA measurement is smaller than the marker size.

where $N$ is the number of events observed passing the inclusive selection, $N_{B G}$ is the number of background events, $\epsilon_{\text {trig }}$ and $\epsilon_{\text {sel }}$ are the trigger and event selection efficiencies, respectively, $1-f_{\xi<10^{-6}}$ accounts for the migration of events with $\xi<10^{-6}$ into the fiducial region and $\mathcal{L}$ is the integrated luminosity corresponding to the data sample. An inelastic cross-section of $\sigma_{\text {inel }}=78.1 \pm$ $2.9 \mathrm{mb}$ is obtained by extrapolating the fiducial crosssection to the full phase space

The LHC experiments have measured $\sigma_{\text {inel }}$ with $\sqrt{s}$ [13] from $\sqrt{s}=6$ to $13 \mathrm{TeV}[15,21,24-28]$. ALICE has measured $\sigma_{\text {inel }}$ at $\sqrt{s}=2.76 \mathrm{TeV}$ [22]. The Pierre Auger Collaboration measured the inelastic p-air cross section at $\sqrt{s}=57 \mathrm{TeV}$ and extracted $\sigma_{\text {inel }}$ using the Glauber model [16]. The measured cross section agrees well with a variety of theoretical predictions and is clearly consistent with the continued rise of the inelastic cross section with centre-of-mass energy, Fig. 10.

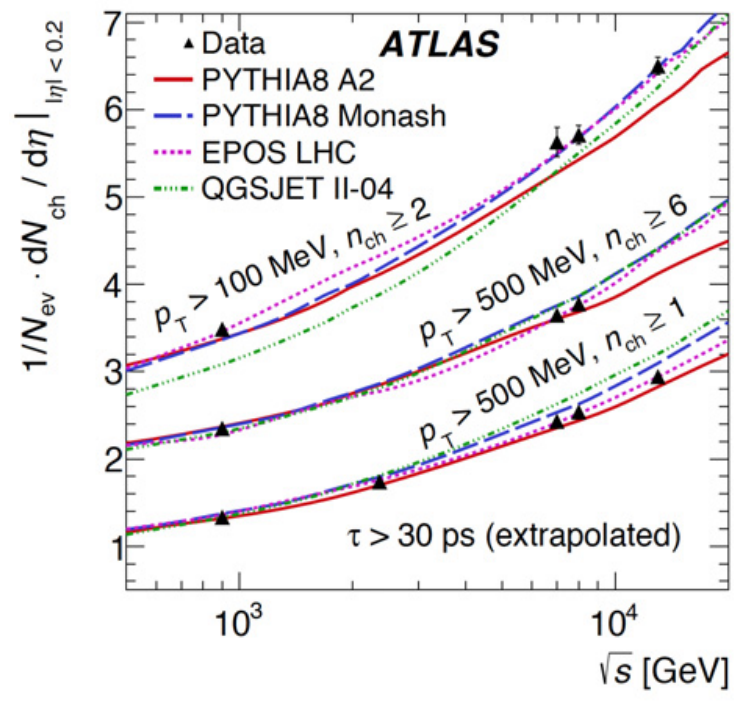

Figure 11. The average primary charged-particle multiplicity in p-p interactions per unit pseudorapidity $\eta$ for $|\eta|<0.2$ as a function of $\sqrt{s}$. The data are shown as black triangles with vertical error bars representing total uncertainty. They are compared to various MC predictions shown as coloured lines.

\section{Charged particle distributions in $\mathrm{p}-\mathrm{p}$ interactions}

ATLAS has performed measurements of distributions of charged particles produced in proton-proton collisions with a centre-of-mass energy $\sqrt{s}$ of of $8 \mathrm{TeV}$ [29] and $13 \mathrm{TeV}$ [30]. The multiplicity, pseudorapidity, and transverse momentum distributions of charged particles are plotted in different kinematic regions and charged particle multiplicity, including measurements of final states at high multiplicity. The results are corrected for detector effects and are compared to the predictions of various Monte Carlo event generator models which simulate the full hadronic final state.

As an example, a plot of the charged particle multiplicity for different transverse momentum and minimum number of charged tracks is given in Fig 11. The values for the other $\mathrm{p}$-p centre-of-mass energies shown in this plot are taken from previous ATLAS analyses $[29,31]$. The value for particles with $\mathrm{p}_{T}>500 \mathrm{MeV}$ at $\sqrt{s}=13 \mathrm{TeV}$ is taken from Ref. [30]. The results have been extrapolated to include charged strange baryons (charged particles with a mean lifetime of $30 \mathrm{ps}<$ $\tau<300$ ps).

For the $13 \mathrm{TeV}$ analysis the data recorded by the ATLAS detector corresponded to an integrated luminosity of $151 \mu b^{-1}$. The particles are required to have a transverse momentum greater than $100 \mathrm{MeV}$ and an absolute pseudorapidity $\eta<2.5$. The measurements performed using proton-proton collisions at $\sqrt{s}=8 \mathrm{TeV}$ were obtained in 2012 in a special run with a small number of interactions per beam crossing (below 0.004) corresponding to an integrated luminosity of $160 \mu \mathrm{b}^{-1}$. In both cases a minimum-bias trigger obtained from the MBTS detector was utilised to select a data sample.

For both studies, the charged-particle multiplicity, its dependence on transverse momentum and pseudorapidity as well as the dependence of the mean transverse momentum on multiplicity are measured in events 
containing at least two charged particles satisfying the above kinematic criteria. The results are corrected for detector effects and compared to the predictions from several Monte Carlo event generators.

\section{Some implications of ATLAS hadronic physics results for cosmic ray physics}

The hadronic physics results from ATLAS shows a very good, in the case of EPOS-LHC, to satisfactory agreement with the various standard Monte Carlo models used in the particle and astroparticle physics arenas. However, all of the models are at least broadly descriptive of the observed ATLAS hadronic physics program data. The ATLAS experiment has recorded hadronic data over the last few years at a centre-of-mass energy that is equivalent to a cosmic ray proton energy of $\sim 10^{17} \mathrm{eV}$ - beyond the knee region $\left(\sim 4 \times 10^{15} \mathrm{eV}\right)$. Consequently, we can rule out the hypothesis that the knee in the cosmic ray spectrum is due to the presence of some strong indication of new hadronic physics threshold, where some $20 \%$ of the cosmic ray primary energy would need to be transferred to invisible channels. Such new physics is not indicated in the hadronic data obtained from ATLAS and CMS.

The hadronic results from ATLAS and other LHC detectors such as CMS and LHCf are extremely useful in refining the models used in high-energy air shower physics reducing the required extrapolation factors to a factor of $\sim 40$. Possible future Collider results with p-O (oxygen) or $\mathrm{p}-\mathrm{N}$ (Nitrogen) would be desirable to extend this program. An example of the use of LHC data in the improvement of our understanding of high energy cosmic ray physics is provided by measurements of the inelastic cross-section by ATLAS, CMS, TOTEM and Auger. These measurements have indicated a slower energy rise of the inelastic crosssection than was previously predicted by some popular models of the hadronic physics of air showers utilized by cosmic ray physicists. This new input lead to a reduction in the p-Air cross-section used in the models resulting in an average deepening of the extended air shower maximum in the atmosphere. A consequence of this finding was that the estimation of the composition of primary cosmic rays moved towards heavier elements as the energy of the cosmic ray primary energy increases.

More recently we have seen that information on hadronic physics doesn't only flow from Collider to Cosmic ray Physics. For example, the measurement of the inelastic p-N - and subsequent estimate of the p-p crosssection at $\sqrt{s}=57 \mathrm{GeV}$ - performed by Auger, is also a useful tool in assessing various hadronic models at LHC energies. Indeed, it seems that some models, for example EPOS 1.99 (and version above), normally utilized largely for studies of cosmic ray air showers, are proving to be a valuable complement to the models traditionally used for studies at Colliders.

\section{The possible use of ATLAS as an underground cosmic muon observatory}

Underground measurements, made by Collider detectors, of multiplicities, or charge ratios of muons produced by cosmic rays in the atmosphere, were first published by

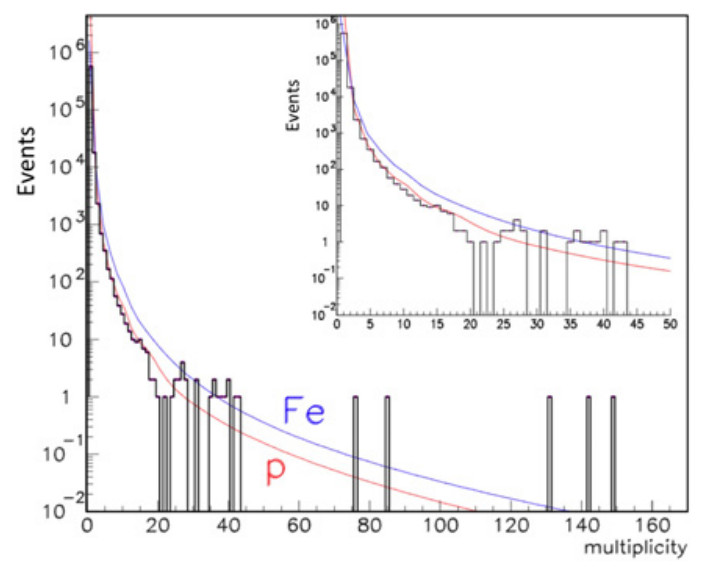

Figure 12. Multiplicity distribution of muons in the TPC compared to CORSIKA simulations for $\mathrm{p}$ and Fe as primary particle.

the LEP experiments ALPHA, DELPHI and L3 [32] that together formed the Cosmo-LEP Collaboration.

In particular, multi-muon events where the muon tracks were parallel and grouped together, called muon bundles, were first highlighted by the Cosmo-LEP activity. They found that muon bundles with very large muon multiplicities measured underground were not described by the then current MC generators. In fact, this was the only LEP result that did not agree with Standard Model expectations! Even the combination of extreme assumptions of highest measured flux value and pure iron spectrum, failed to describe the abundance of high multiplicity events. An example of the discrepancy between measurement and Monte Carlo prediction in this arena is shown in Fig. 12. At the LHC ALICE has already reported similar measurements using their ACORDE subdetector, as shown in Fig. 13 [33]. The horizontal detection area for the ALICE ACORDE cosmic ray detector of $12.5 \mathrm{~m}^{2}$ is defined by the ALICE TPC. Again, it can be seen that the MC simulations are struggling to explain the highly multiplicity tail of the muon-bundle multiplicity plot. The ATLAS muon system covers a cylinder $20 \mathrm{~m}$ in diameter and $25 \mathrm{~m}$ long with a horizontal cross section of approx. $500 \mathrm{~m}^{2}$. It consists of roughly $12000 \mathrm{~m}^{2}$ of total detector area and roughly 1 million channels of data readout Several types of muon detectors but $30 \mathrm{~mm}$ diameter straw tubes are the main element. Thus, ATLAS provides an unprecedented capability for precision cosmic muon measurement within a magnetic field.

The ACME group of the ATLAS collaboration [34] is preparing a proposal to use the ATLAS-LHC detector in conjunction with a surface array of scintillator detectors, as a facility to study comic ray muon physics. Operating ATLAS in this mode will provide unique data on multi-muon production in cosmic ray air showers in a particularly interesting energy regime. Together with the simultaneous and complementary measurement of the electromagnetic structure of the air showers, ACME will be able to determine the composition of cosmic ray primaries over the energy range $10^{14} \mathrm{eV}$ to a few times $10^{16} \mathrm{eV}$. In addition, it will provide a sensitive instrument for studying the structure of cosmic ray air showers and to search for new physics in the form of exotic phenomena such as Centauro and anti-Centauro events, 


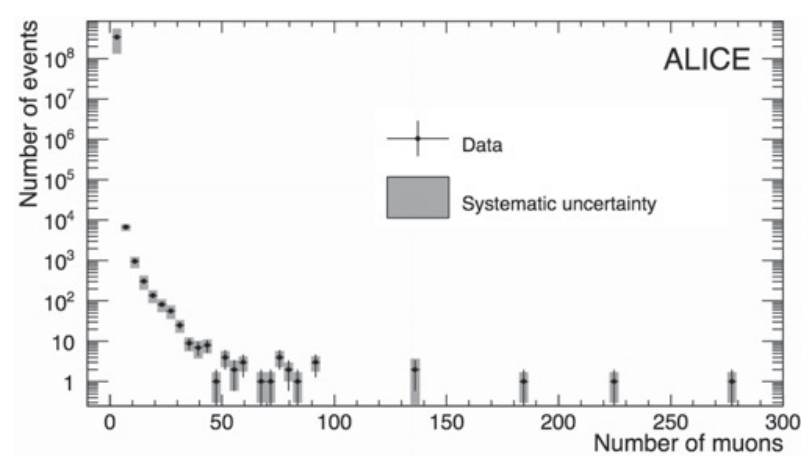

Figure 13. Muon multiplicity distribution of the whole sample of data (2010-2013) corresponding to 30.8 days of data taking.

and, significantly extend the study of the high multiplicity muon excess previously observed, for example, with the Cosmo-LEP detectors. Lastly, the surface array can be used to flag large cosmic ray showers that may contribute backgrounds to exotic physics processes produced in ATLAS collisions.

\section{Conclusion}

Current models of hadronic interactions are broadly descriptive of the observed ATLAS hadronic physics program data. The ATLAS data recorded over the last year or so were taken at a centre-of-mass energy that is equivalent to a cosmic ray proton energy $10^{17} \mathrm{eV}$ just beyond the knee region $\left(10^{15} \mathrm{eV}\right)$. Consequently, we can rule out the hypothesis that the knee in the cosmic ray spectrum is due to the effect of some new hadronic physics threshold, where some $20 \%$ of the cosmic ray primary energy would need to be transferred to invisible channels. Future ATLAS running at $13 \mathrm{TeV}$ will allow us to further refine our hadronic interaction models. Importantly the turn on of the AFP detector will enable important studies of single and double diffraction, a key input to our overall understanding of hadronic physics at the LHC. Last, but not least, the use of the ATLAS detector as a high precision cosmic-muon observatory would enable the LHC to contribute directly to our understanding of high energy cosmic ray physics across the knee region of the cosmic ray energy spectrum.

\section{References}

[1] K. Kotera and A.V. Olinto, Ann. Rev. Astron. Astrophys. 49, 119 (2011)

[2] A.M. Hillas, Ann. Rev. Astron. Astrophys. 22, 425 (1984)

[3] J. Blümer, R. Engel, and J. R. Hörandel, Prog. Part. Nucl. Phys. 63, 293 (2009)

[4] V. Berezinsky, A.Z. Gazizov, and S.I. Grigorieva, Phys. Rev. D 74, 043005 (2006)

[5] D. Allard, E. Parizot, E. Khan, S. Goriely, and A.V. Olinto, Astron. Astrophys. 443, L29 (2005)

[6] K. Greisen, Phys. Rev. Lett. 16, 748 (1966); G. T. Zatsepin and V. A. Kuzmin J. Exp. Theor. Phys. Lett. 4, 78 (1966)
[7] J. Abraham, Phys. Rev. Lett. 104, 091101 (2010); D. Allard, Astropart. Phys. 39-40, 33 (2012); P. Abreu et al., JCAP 1302, 026 (2013)

[8] K.-H. Kampert and M. Unger, Astropart.Phys. 35, 660 (2012)

[9] A. Sidoti et al., ATLAS Collaboration, JINST 9, C10020 (2014)

[10] S. Abdel Khalek et al., ALFA/ ATLAS Collaboration, JINST 11, P11013 (2016)

[11] S. Grinstein, AFP/ATLAS Collaboration, Nucl. Part. Phys. Proc. 273-275, 1180 (2016)

[12] M. Aabound et al., The ATLAS Collaboration, Phys. Lett. B 761, 158 (2016)

[13] K.A. Olive, et al., Particle Data Group, in: Review of Particle Physics, Chin. Underground measurements, made by Collider detectors, of multiplicities or charge ratios of muons produced by cosmic. C $\mathbf{3 8}$, 090001 (2014)

[14] G. Antchev, et al., TOTEM Collaboration, Nucl. Phys. B 899, 527 (2015)

[15] G. Antchev, et al., TOTEM Collaboration, Europhys. Lett. 101, 21002 (2013)

[16] P. Abreu, et al., Pierre Auger Collaboration, Phys. Rev. Lett. 109, 062002 (2012)

[17] G. Aielli, et al., ARGO-YBJ Collaboration, Phys. Rev. D 80 (2009) 092004

[18] M. Honda, et al., Phys. Rev. Lett. 70, 525 (1993)

[19] R.M. Baltrusaitis, et al., Phys. Rev. Lett. 52, 1380 (1984)

[20] M. Aaboud et al., ATLAS Collaboration, Phys. Rev. Lett. 117, 182002 (2016)

[21] G. Antchev, et al., TOTEM Collaboration, Phys. Rev. Lett. 111, 012001 (2013)

[22] B. Abelev et al., ALICE Collaboration, Eur. Phys. J. C 73, 2456 (2013)

[23] LHCb Collaboration, R. Aaij et al., JHEP 02, 129 (2015)

[24] M. Aaboud et al., ATLAS Collaboration, Nucl. Phys. B 889, 486 (2014)

[25] G. Antchev et al., TOTEM Collaboration, Eur. Phys. J. C76, 661 (2016)

[26] M. Aaboud et al., ATLAS Collaboration. Phys. Lett. B 761, 158 (2016)

[27] G. Aad, ATLAS Collaboration, Nature Commun. 2, 463 (2011)

[28] S. Chatrchyan, CMS Collaboration, Phys. Lett. B 722, 5 (2013)

[29] M. Aaboud et al., ATLAS Collaboration, Eur. J. Phys. C76, 403 (2016)

[30] M. Aaboud et al., ATLAS Collaboration, Eur, J. Phys. C76, 502 (2016)

[31] G. Aad et al., ATLAS Collaboration, New J. Phys. 13, 053033 (2011)

[32] C. Avati et al., CORAL Collaboration, CERN/2001003, SPSC/P231 (2001) and references therein

[33] J. Adam et al., ALICE Collaboration, JCAP 1601, 032 (2016)

[34] J.L. Pinfold, Proceedings of the 13th ICATPP Conference, Villa Olmo, Como, Italy, 3-7 October 2011, DOI: http://dx.doi.org/10.1142/ 9789814405072_0158 